\title{
Oxidation threshold in silicon etching at cryogenic temperatures
}

\author{
T. Tillocher, P. Lefaucheux, X. Mellhaoui, R. Dussart, P. Ranson* \\ GREMI-Polytech'Orléans, 14 rue d'Issoudun, \\ BP 6744, 45067 Orléans cedex 2, France \\ M. Boufnichel \\ STMicroelectronics, 16 rue Pierre et Marie Curie, \\ BP 7155, 37071 Tours cedex 2, France \\ L. J. Overzet \\ The Plasma Applications Laboratory, \\ University of Texas at Dallas, Richardson, Texas 75083-0688
}

(Dated: July 13, 2005)

\begin{abstract}
In silicon etching in $\mathrm{SF}_{6} / \mathrm{O}_{2}$ plasmas, an oxidation threshold appears when the oxygen content is high enough. This threshold can be reached for lower oxygen proportions when the substrate is cooled down to cryogenic temperatures. In this paper, we present a mass spectrometry study of this oxidation threshold in different experimental conditions (temperature, source, self-bias). A model, describing the oxygen coverage as a function of the parameters mentioned above, is proposed to interpret these results.
\end{abstract}

PACS numbers:

*Electronic address: pierre.ranson@univ-orleans.fr 
The cooling of patterned silicon wafers is necessary to etch high aspect ratio structures in $\mathrm{SF}_{6} / \mathrm{O}_{2}$ plasmas: this is the so called cryogenic process. At temperatures as low as $-100^{\circ} \mathrm{C}$, a $\mathrm{SiO}_{x} \mathrm{~F}_{y}$ passivation layer is formed on the sidewalls and acts as an etch inhibitor [1]. Since ion bombardment prevents its formation at the bottom of the patterns, anisotropic etching can then be achieved.

The formation of a similar layer has been previously reported by several authors during the etching of maskless silicon wafers [2-5]. They worked at room temperature in $\mathrm{CF}_{4} / \mathrm{O}_{2}$ and $\mathrm{SF}_{6} / \mathrm{O}_{2}$ gas mixtures. Their XPS measurements confirmed the presence of silicon, oxygen and fluorine in this layer. It also enabled the computation of its stoichiometry and its thickness as a function of the $\mathrm{O}_{2}$ content in the plasma. As the $\mathrm{O}_{2}$ proportion was increased in percentage, the surface was increasingly oxidized and the stoichiometry gradually changed from $\mathrm{SiF}_{2}$ without oxygen, to $\mathrm{SiOF}_{2}$ at middle proportions and finally to $\mathrm{SiOF}[2,3]$. However, the film thickness did not have a monotonic evolution: it first decreased with increasing $\mathrm{O}_{2}$ percentage and then began to increase again. The thickness of the $\mathrm{SiO}_{x} \mathrm{~F}_{y}$ layer, or in other words the level of oxidation, directly impacted the etch rate as well. Thus, above an $\mathrm{O}_{2}$ proportion threshold, the layer became thick enough to strongly reduce the etch rate. This agreed well with the studies of Puech et al. who showed the existence of such an etch threshold at cryogenic temperatures for low oxygen concentrations [6, 7]. They also noticed that increasing either the temperature or the self-bias makes the threshold occur at higher oxygen contents.

In this article, we present our studies of the threshold of oxidation using bare silicon wafers and mass spectrometry as a function of the $\mathrm{O}_{2}$ proportion at room temperature and especially at low temperatures. We also highlight the role of both source power and chuck self-bias ; the increase of which can shift the threshold to higher oxygen proportions. These results are then discussed with the help of a short model modified from that in reference [8].

An industrial ICP (Inductively Coupled Plasma) reactor (Alcatel $601 \mathrm{E}$ ) only dedicated to cryogenic etching has been utilized for the experiments detailed in this letter. $\mathrm{SF}_{6} / \mathrm{O}_{2}$ plasmas are created in the alumina tube of the source. It can be powered up to $3 \mathrm{~kW}$. The maximum gas flows are $1000 \mathrm{sccm}$ for $\mathrm{SF}_{6}$ and $70 \mathrm{sccm}$ for $\mathrm{O}_{2}$. A magnetic field of 15 Gauss confines the plasma in both the source and the diffusion chamber. The reactor is equipped with an electrostatic chuck cooled with liquid nitrogen and warmed up with a heating element. It enables us to regulate the temperature between $-150^{\circ} \mathrm{C}$ and $+40^{\circ} \mathrm{C}$ with 
high accuracy. Helium enhances the thermal contact between the substrate and its holder. This chuck is independently rf-biased with a $500 \mathrm{~W}$ power supply, which is voltage-regulated. A Quadrupole Mass Spectrometer (Balzers QMS 200) is mounted $5 \mathrm{~cm}$ above the bottom of the diffusion chamber [9]. Molecules are extracted from the plasma through a homemade limiter that reduces the detection of molecules recombined after their entrance in the spectrometer. Two signals of interest for our experiments are $\mathrm{SiF}_{3}^{+}$and $\mathrm{F}^{+}$. Indeed, the $\mathrm{SiF}_{3}^{+}$line dominantly comes from the fragmentation of $\mathrm{SiF}_{4}$ which is the main etch product; because $\mathrm{Si}_{2} \mathrm{~F}_{6}$ is produced in negligible quantities [3]. Thus the $\mathrm{SiF}_{3}^{+}$signal is reasonably proportional to the etch rate. The interpretation of the $\mathrm{F}^{+}$signal is not so obvious since it can result from the fragmentation of many species in the ionizer. Consequently, it does not only reflect the concentration of $\mathrm{F}$ radicals.

Experiments were first carried out at $3 \mathrm{~Pa}, 700 \mathrm{~W}$ source power and no bias. In this case, the $\mathrm{O}_{2} / \mathrm{SF}_{6}$ (feed gas flow ratio) percentage was varied from 0 to $35 \%$. The pressure in the spectrometer was approximately $7 \cdot 10^{-6}$ mbar. 6" bare or $\mathrm{SiO}_{2}$ coated silicon wafers have been used. They are $400 \mu \mathrm{m}$ thick and (100) oriented. The $\mathrm{SiO}_{2}$ coating, if present, is nearly $0.8 \mu \mathrm{m}$ thick.

Fig. 1a shows the $\mathrm{SiF}_{3}^{+}$peak intensity as a function of the oxygen content for processing bare silicon wafers at room temperature and $-85^{\circ} \mathrm{C}$ and $\mathrm{SiO}_{2}$ coated wafer at room temperature. In this last case, the signal is quite small because $\mathrm{SiO}_{2}$ is etched much slower than silicon. It also weakly increases with oxygen proportion. For the etching on a bare silicon wafer at ambient temperature, the $\mathrm{SiF}_{3}^{+}$signal first increases up to maximum at 5\%. Then, it slowly decreases down to a very sharp drop at $25 \%$. This is the threshold where oxidation begins to dominate etching. This is clearly shown on fig. 1a: the $\mathrm{SiF}_{3}^{+}$signal becomes as low as that for $\mathrm{SiO}_{2}$ hence the etching is strongly reduced. This is in agreement with the results in ref $[2,3,5,10]$.

At $-85^{\circ} \mathrm{C}$, the trend is slightly different: the threshold is reached for an oxygen percentage around $2.5 \%$. Then, the $\mathrm{SiF}_{3}^{+}$signal increases more quickly than for the $\mathrm{SiO}_{2}$ masked wafer. This suggests that the layer is not as strong as an oxide film: as this layer already contains fluorine, it can be etched more easily and rapidly. Moreover, the $\mathrm{SiF}_{3}^{+}$signal maximum at $-85^{\circ} \mathrm{C}$ is not as high as it is at ambient temperature. This probably does not mean that the fluorine reactivity with silicon is reduced at cryogenic temperatures, but that less $\mathrm{SiF}_{4}$ is desorbed. This is in agreement with a passivation mechanism detailed in ref [9] : O radicals 
can then react with $\mathrm{SiF}_{x}$ sites and create a passivation layer. This is more efficient when the substrate temperature is lower, as can be inferred from fig. 2a. The oxidation threshold quasi linearly increases from $-85^{\circ} \mathrm{C}$ to $0^{\circ} \mathrm{C}$ which suggests that atomic oxygen has a higher sticking coefficient on $\mathrm{SiF}_{x}$ sites at lower temperature. This is consistent with studies which show a higher initial sticking coefficient of molecular oxygen on silicon at cryogenic temperatures $[11,12]$, but we have not been able to find similar references for atomic oxygen adsorption on fluorinated silicon surfaces.

These trends are consistent with the $\mathrm{F}^{+}$peak intensity under the same experimental conditions, displayed on fig. 1b. When the oxidation threshold is reached, etching is strongly reduced and the consumption of fluorine is reduced. As a result the $\mathrm{F}^{+}$signals sharply increase just after the thresholds determined from the $\mathrm{SiF}_{3}^{+}$lines.

The increase of the etch product signal $\left(\mathrm{SiF}_{3}^{+}\right)$at low oxygen concentrations can be explained in terms of $\mathrm{SF}_{6} / \mathrm{O}_{2}$ chemistry. The addition of oxygen in $\mathrm{SF}_{6}$ plasmas promotes fluorine creation $[13,14]$. Such an effect is clearly shown on fig. $1 \mathrm{~b}$ : the $\mathrm{F}^{+}$peak intensity increases with oxygen when the substrate has a $\mathrm{SiO}_{2}$ mask. Indeed, reaction of oxygen with $\mathrm{SF}_{x}$ species reduces their recombination with $\mathrm{F}$ atoms (eq. 1) and also increases $\mathrm{F}$ production (eq. 2).

$$
\begin{gathered}
S F_{x}+O \stackrel{\text { wall }}{\longrightarrow} S O F_{x}(\mathrm{x}=1-4) \\
S F_{x}+O \longrightarrow S O F_{x-1}+F(\mathrm{x}=1-5)
\end{gathered}
$$

The first increase of the etch rate can also be attributed to sulfur atoms which are reported to have a retarding effect on oxidation at room temperature $[2,10]$. For low oxygen contents, sulfur is present on the surface and prevents oxygen atoms from adsorbing. But for higher concentrations, sulfur is no longer efficient [2]. In our case, a very low temperature seems to reduce this effect since the oxygen adsorption probability is increased. This also explains why the $\mathrm{SiO}_{x} \mathrm{~F}_{y}$ film is created for lower percentages of oxygen.

Physical mechanisms have non negligible effects on the oxidation as well. Figure $2 \mathrm{~b}$ shows the oxidation threshold as a function of the source power at a substrate temperature of $-85^{\circ} \mathrm{C}$. The other plasma parameters are not changed. The threshold seems to increase linearly with the power from $700 \mathrm{~W}$ to $2000 \mathrm{~W}$.

The observation is the same on fig. 2c, which displays the oxidation threshold against 
the bias, at the same temperature: we also have a quasi linear evolution. After each of these experiments, columnar microstructures (CMS) had appeared on the substrates. This is an evidence of the formation of a strong passivation layer at high oxygen contents [15]. And these CMS appear for higher oxygen content when the self-bias is increased because oxidation is delayed.

Such results can be interpreted in terms of density of power $\left(\mathrm{P}_{i}\right)$ deposited on the surface by the positive ions expressed as [16]:

$$
P_{i}=\Gamma_{i} E_{i}=\Gamma_{i}\left(\epsilon_{i}+q\left(V_{p}-V_{b}\right)+2 k T_{e}\right)
$$

$\Gamma_{i}$ is the ion flux and is proportional to the ion density which can be increased with the source power. The second term, $\mathrm{E}_{i}$, is composed of the ionization energy $\epsilon_{i}$, the energy acquired in the sheath $q\left(V_{p}-V_{b}\right)$ and the electron energy. The $q\left(V_{p}-V_{b}\right)$ quantity can be controlled with the bias $\mathrm{V}_{b}$. Consequently, increasing either the source power or the selfbias contributes to increase either the ion flux or the ion energy $\left(E_{i}\right)$ hence the density of power transferred to the surface by ions and especially to the $\mathrm{SiO}_{x} \mathrm{~F}_{y}$ layer. This is consistent with the disappearance of the passivation layer when the substrate is heated: the ion bombardment effectively warms up the surface by supplying enough energy to desorb the film [9]. Consequently, this removal mechanism is different from sputtering.

We now consider a modified version of the model proposed by Mogab et al. and also used by Campo et al $[2,3,8]$. It should take into account the mechanisms involved at cryogenic temperatures:

$$
\frac{d \theta_{O}}{d t}=k_{a}(T)\left(1-\theta_{O}\right) N_{O}-k_{d} \theta_{O} N_{F}-k_{i}\left(E_{i}\right) \theta_{O} N_{i}
$$

$\theta_{o}$ is the oxygen coverage of the silicon, $\mathrm{k}_{a}$ is the adsorption of oxygen on silicon rate constant and $\mathrm{N}_{o}$ the oxygen concentration. $\mathrm{k}_{d}$ is the rate constant associated to the desorption of oxygen by the etching and $\mathrm{N}_{f}$ the fluorine density. This equation illustrates the fact that oxygen and fluorine are in competition for the adsorption sites on silicon through the first two terms when no bias is applied to the substrate. In such a case and at steady state, the oxygen coverage is given by:

$$
\theta_{O}=\frac{1}{1+\frac{k_{d} N_{F}}{k_{a} N_{O}}}
$$

The limiting case, $\theta_{o}=1$, is obtained when oxidation dominates etching $\left(\mathrm{k}_{d} \mathrm{~N}_{F} \ll \mathrm{k}_{a} \mathrm{~N}_{O}\right)$ 
hence for high oxygen concentrations or for higher values of $k_{a}$. This is certainly achieved at very low temperatures since the oxidation is then more efficient (fig 2.a). That's why we consider that $\mathrm{k}_{a}$ depends on the temperature. The last term in eq. 5 represents the ion contribution in the removal of the passivation layer. The rate constant, $\mathrm{k}_{i}$, which depends on the ion energy $\mathrm{E}_{i}$, is used to describe the desorption of oxygen by ion bombardment and $\mathrm{N}_{i}$ is the ion density. $\theta_{o}$ now becomes :

$$
\theta_{O}=\frac{1}{1+\frac{k_{d} N_{F}+k_{i} N_{i}}{k_{a} N_{O}}}
$$

An increase in the ion contribution can cause the oxygen coverage to decrease. As it has been previously shown, this can be achieved by increasing either the source power or the bias. This is in good agreement with the experimental results. As oxygen plays a key role in the formation of the passivation layer, we can assume that eq. 4 can also be used to describe its behavior.

These experiments have given new elements about the oxidation of silicon. $\mathrm{SiO}_{x} \mathrm{~F}_{y}$ films grow when the $\mathrm{O}_{2}$ percentage is sufficiently large. An oxidation threshold occurs for lower oxygen proportions when the substrate is cooled. This threshold is reached for higher $\mathrm{O}_{2}$ contents when either the source power or the self-bias is increased. We have presented a model which is in good agreement with both our results and a passivation mechanism for cryoetching proposed in ref [9].

The authors acknowledge STMicroelectronics (Tours, France) for helpfull discussions. This work is supported by STMicroelectronics and by the French Governement.

[1] R. Dussart, M. Boufnichel, G. Marcos, P. Lefaucheux, A. Basillais, R. Benoit, T. Tillocher, X. Mellhaoui, H. Estrade-Szwarckopf, and P. Ranson, J. Micromech. Micromech 14, 190 (2004).

[2] A. Campo, C. Cardinaud, and G. Turban, J. Vac. Sci. Techolog. B 13, 235 (1995).

[3] A. Campo, C. Cardinaud, and G. Turban, Plasmas Sources Sci. Technolog. 4, 398 (1995).

[4] J. H. Thomas and J.-S. Maa, Appl. Phys. Lett. 43, 859 (1983).

[5] G. S. Oehrlein, S. W. Robey, and J. L. Linstrm, Appl. Phys. Lett. 52, 1170 (1988).

[6] J. W. Bartha and M. P. a. M. J. Greschner, Microelectron. J. p. 453 (1995). 
[7] M. Puech and P. Maquin, Appl. Surf. Sci. 100/101, 579 (1996).

[8] C. J. Mogab, A. C. Adams, and D. L. Flamm, J. Appl. Phys. 49, 3796 (1978).

[9] X. Mellhaoui, R. Dussart, T. Tillocher, P. Lefaucheux, and P. Ranson, J. Appl. Phys. (2005), submitted.

[10] K. Ninomiya, K. Suzuki, S. Nishimatsu, and O. Okada, J. Appl. Phys. 62, 1459 (1987).

[11] J. Dabroski and H.-J. Mssig, Silicon Surfaces and Formation of Interfaces (World Scientific, 2000).

[12] T. Engel, Surf. Sci. Rep. 18, 91 (1993).

[13] K. R. Ryan and I. C. Plumb, Plasma Chem. Plasma Process. 8, 263 (1988).

[14] R. d'Agostino and D. L. Flamm, J. Appl. Phys. 52, 162 (1989).

[15] R. Dussart, X. Mellhaoui, T. Tillocher, P. Lefaucheux, M. Volatier, C. Socquet-Clerc, P. Brault, and P. Ranson, J. Phys. D (2005), submitted.

[16] M. A. Lieberman and A. J. Lichtenberg, Principles of plasma discharges and materials processing (Wiley-interscience, 1994). 

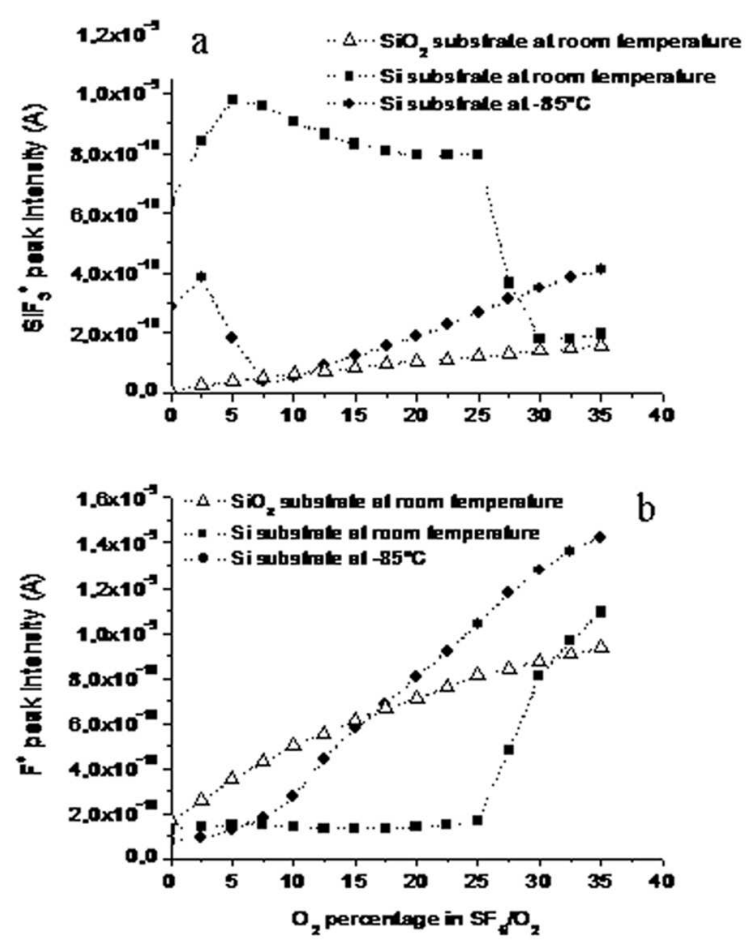

FIG. 1: (a) $\mathrm{SiF}_{3}^{+}$signal peak intensity versus the oxygen percentage in three cases for $3 \mathrm{~Pa}, 700 \mathrm{~W}$ and without bias (b) $\mathrm{F}^{+}$signal peak intensity versus the oxygen percentage in the same conditions 

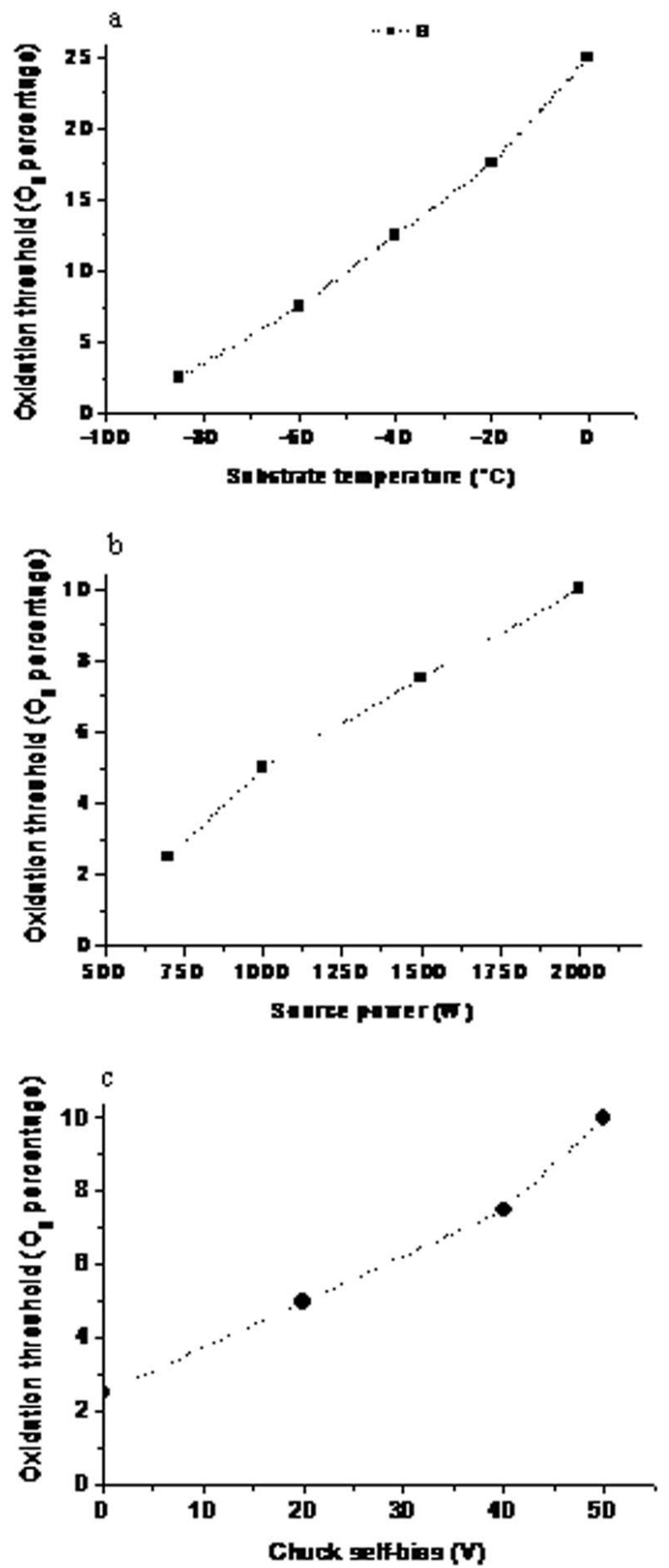

FIG. 2: (a) Oxidation threshold versus the substrate temperature for $3 \mathrm{~Pa}, 700 \mathrm{~W}$ and without bias (b) Oxidation threshold versus the source power for $3 \mathrm{~Pa},-85^{\circ} \mathrm{C}$ and no bias (c) Oxidation threshold versus the chuck self-bias for $3 \mathrm{~Pa},-85^{\circ} \mathrm{C}$ and $700 \mathrm{~W}$ 